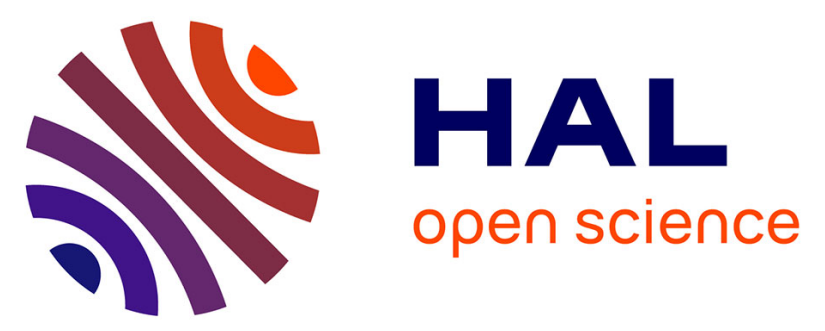

\title{
A study on local photometric models and their application to robust tracking
}

Michèle Gouiffès, Christophe Collewet, Christine Fernandez-Maloigne, A. Trémeau

\section{- To cite this version:}

Michèle Gouiffès, Christophe Collewet, Christine Fernandez-Maloigne, A. Trémeau. A study on local photometric models and their application to robust tracking. Computer Vision and Image Understanding, 2012, 116 (8), pp.896-907. 10.1016/j.cviu.2012.04.002 . hal-00726513

HAL Id: hal-00726513

https://hal.inria.fr/hal-00726513

Submitted on 30 Aug 2012

HAL is a multi-disciplinary open access archive for the deposit and dissemination of scientific research documents, whether they are published or not. The documents may come from teaching and research institutions in France or abroad, or from public or private research centers.
L'archive ouverte pluridisciplinaire $\mathbf{H A L}$, est destinée au dépôt et à la diffusion de documents scientifiques de niveau recherche, publiés ou non, émanant des établissements d'enseignement et de recherche français ou étrangers, des laboratoires publics ou privés. 


\title{
A study on local photometric models and their application to robust tracking.
}

\author{
M. Gouiffès ${ }^{(1)}$, C. Collewet $^{(2)}$, C. Fernandez ${ }^{(3)}$, A. Trémeau ${ }^{(4)}$ \\ (1) michele.gouiffes@ief.u-psud.fr \\ Institut d'Electronique Fondamentale CNRS UMR 8622 Université \\ Paris-Sud 11, 91405 ORSAY cedex \\ (2) IRSTEA and INRIA, Rennes, France \\ (3) XLIM, Université de Poitiers, France \\ (4) Laboratoire Hubert Curien, St Etienne, France
}

\begin{abstract}
Since modeling reflections in image processing is a difficult task, most computer vision algorithms assume that objects are Lambertian and that no lighting change occurs. Some photometric models can partly answer this issue by assuming that the lighting changes are the same at each point of a small window of interest. Through a study based on specular reflection models, we explicit the assumptions on which these models are implicitly based and the situations in which they could fail.

This paper proposes two photometric models, which compensate for specular highlights and lighting variations. They assume that photometric changes vary smoothly on the window of interest. Contrary to classical models, the characteristics of the object surface and the lighting changes can vary in the area being observed. First, we study the validity of these models with respect to the acquisition setup: relative locations between the light source, the sensor and the object as well as the roughness of the surface. Then,
\end{abstract}


these models are used to improve feature points tracking by simultaneously estimating the photometric and geometric changes. The proposed methods are compared to well-known tracking methods robust to affine photometric changes. Experimental results on specular objects demonstrate the robustness of our approaches to specular highlights and lighting changes.

Keywords: Computer vision, robust feature point tracking, local photometric models.

\section{Introduction}

Computer vision has recently emerged in many fields such as mobile robotics [1], visual inspection, in surgical, industrial, agricultural, spatial or underwater domains [2], i.e. in various natural environments. For such

practical applications, one of the crucial problems lies in the robustness of the low level algorithms with respect to some critical acquisition conditions: blurred images, acquisition noise, illumination changes, reflections. High level algorithms such as 3D reconstruction, active vision or visual servoing can be efficiently improved by increasing the robustness of the spatial and temporal matching process.

This paper addresses more precisely the problem of robust feature tracking with respect to lighting changes and specular highlights. The issue can be tackled by extracting salient features in the image, such as edges [3, 4], corners [5], lines [6]. It becomes far more complicated in most natural environments when only points are likely to be detectable. However, tracking those features is not trivial since it relies on the luminance of the neighbor pixels which are highly sensitive to photometric variations. The seminal 
works in the domain are due to Lucas and Kanade $[7,8]$ (KLT) who assume the luminance constancy [9] in order to compute the translation motion of each point.

This work has been extended to more comprehensive motion models: affine [10], quadratic [11] and homographic [12, 13], and is still considered to be powerful [14]. The tracking of planar patterns can also be implemented by an efficient second order minimization technique (ESM) [15].

However, most methods assume that the luminance remains constant between two successive frames, which is not true. Indeed, most surfaces are not Lambertian and lighting conditions are mostly variable during an image sequence. Hager and Belhumeur [16] propose to acquire an image database of the scene under several illuminations and to use these data to improve the tracking. Although efficient, this method requires a prior learning step, which can be seen as too restrictive. Alternatively, one can either use a simple local photometric normalization as in [17] or compute a photometric model which properly fits the luminance variations in small areas of the image as $[18,19,20,21]$ for optical flow, [22] for object recognition, or [23] for indexing. In [24], the tracker compensates for affine illumination changes. More recently in [25], the authors compute arbitrary illumination changes on a large planar area by using an ESM algorithm. The main difficulty of the illumination compensation is to balance the trade-off between complexity and adequacy with the real illumination changes. Moreover, these models are based on several assumptions which have not been clearly defined yet.

Our first contribution is to clearly explain the modeling of luminance changes due to lighting and geometry, by analyzing some widely used specu- 
lar reflection models $[26,27]$. We particularly focus on two local approximations: the first one is well suited for specular highlights and lighting changes when small areas are concerned; the second one remains apropriate for larger windows.

The second contribution of the paper consists in studying the validity of the proposed models, which is related to the object parameters and the acquisition setup. Note that the surfaces are considered to be differentiable, which is a reasonable assumption for: 1) small surfaces such as points, 2) industrial applications where manufactured objects and natural surfaces have to be manipulated.

Finally, we propose a KLT-like tracker. Compared to most efficient KLTlike trackers, it leads to the best results in terms of the number of points correctly tracked, residuals and location errors.

This article is organized as follows. Section 2 focuses on the general modeling of luminance changes, especially in the case of specular reflections and lighting variations. Then, Section 3 deals with the local illumination models used for temporal matching, and then details the two photometric models proposed. The theoretical validity of the photometric models is studied in Section 4 by considering several specific configurations on the viewing geometry and the surface properties. Section 5 details some of the existing trackers, regarding the illumination model on which they are based, and explains the proposed methods. The relevance of the present work is validated through experimental results in Section 6. 


\section{Modeling of luminance changes}

The description of the luminance is detailed hereafter, it is based upon simplified physical models largely used in image analysis. For image synthesis, some more elaborated BRDF are considered. This study focuses on the luminance changes occurring between two successive images of the same scene.

A uniform lighting and a point light source are considered, which is well suited for our targeted applications in industrial contexts or outdoor scenes where the is considered as a point light source. More generally, each point in the scene is assumed to be locally lighted by a dominant point light source.

First, let us introduce our notations (see Figure 1). Let $P$ be a point of the object being observed, and $\mathbf{V}$ and $\mathbf{L}$ the viewing and lighting directions respectively, which form the angles $\theta_{r}$ and $\theta_{i}$ with the normal $\mathbf{n}$ in $P$. $\mathbf{B}$ is the bisecting line between $\mathbf{V}$ and $\mathbf{L}$, and forms an angle $\rho$ with $\mathbf{n}$. $f$ and $f^{\prime}$ are two successive images of the same scene. $P$ projects in image $f$ into $p$ of coordinates $\left(x_{p}, y_{p}\right)$ and into $p^{\prime}$ of coordinates $\left(x_{p}^{\prime}, y_{p}^{\prime}\right)$ in the image $f^{\prime}$ after a relative motion between the camera, the scene and the light sources. More generally, the prime symbol refers to the parameters in $f^{\prime}$.

\subsection{The luminance in the CCD plane}

The luminance $f(p)$ results from the integration of the radiance $\mathcal{L}_{P}(\lambda)$ emitted by $P$ w.r.t the wavelength $\lambda$. Since the radiance is the product of the illuminant spectrum $\mathcal{E}_{P}(\lambda)$ with the material reflectance $\mathcal{R}_{P}(\lambda)$ then

$$
f(p)=k_{c} \int_{\lambda_{\min }}^{\lambda_{\max }} \mathcal{S}(\lambda) \mathcal{L}_{P}(\lambda) d \lambda=k_{c} \int_{\lambda_{\min }}^{\lambda_{\max }} \mathcal{S}(\lambda) \mathcal{E}_{P}(\lambda) \mathcal{R}_{P}(\lambda) d \lambda
$$




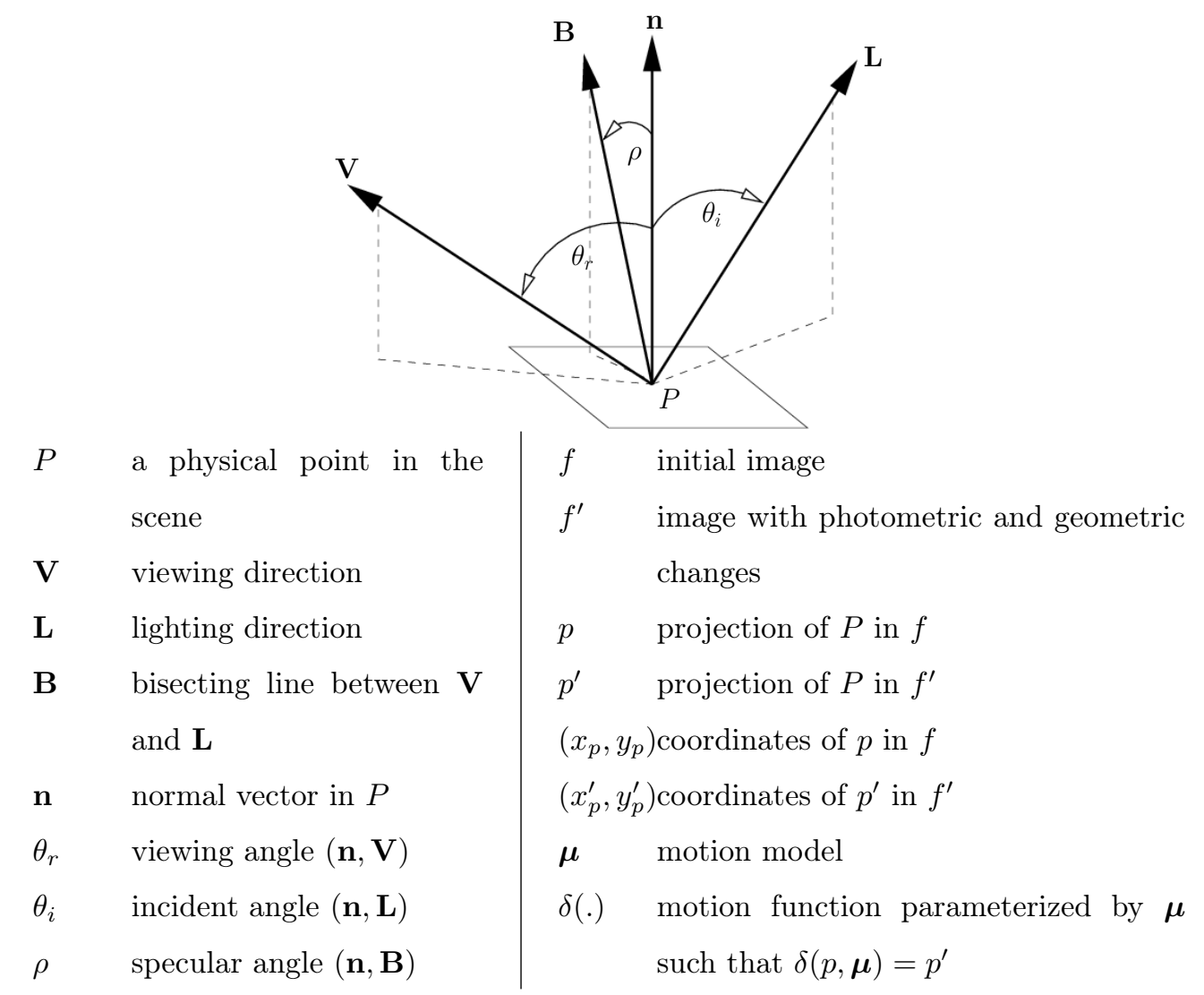

Figure 1: Notations involved in the reflection description.

where $\mathcal{S}(\lambda)$ describes the spectral sensitivity of the sensor and $k_{c}$ is a scalar which does not depend on the wavelength $\lambda$ but only on the optical geometry such as the focal distance and the aperture.

The reflectance is generally assumed to be diffuse or Lambertian [28], i.e it does not depend on $\theta_{r}$ and it is a function of the body reflection called $\mathcal{R}_{P}^{b}(\lambda)$.

However, most surfaces reflect light in a specular manner, not only in a diffuse way. For example, the Phong-Blinn model $[26,29]$ describes the 
reflectance of specular surfaces in the following heuristic way, for $\theta_{i}(P) \in$ $\left[-\frac{\pi}{2}, \frac{\pi}{2}\right]:$

$$
\mathcal{R}_{P}(\lambda)=k_{d}(P) \mathcal{R}_{P}^{b}(\lambda) \cos \theta_{i}(P)+k_{s}(P) \cos ^{r}(\rho(P))+k_{a}(P)
$$

The first term is the Lambertian contribution while the second one is the specular component, where the scalar $r$ is inversely proportional to the roughness of the surface and $k_{s}, k_{a}$ are the specular and ambient gains in $P$. Although empirical, this model is largely used, because of its simplicity, and its validity for various types of materials, whether they are rough or smooth. Note that the Torrance-Sparrow model [27], is also widely used. In both models, the specular term reaches its maximum value for $\rho(P)=0$, that is when $\mathbf{B}$ coincides with $\mathbf{n}$. In the remainder of the paper, this specular term in $P$ is noted $h(P)$.

\subsection{The modeling of the luminance in the image}

Consider $\mathcal{M}_{P}(\lambda)=\mathcal{S}(\lambda) \mathcal{E}_{P}(\lambda)$ in (1). When the sensor has a linear response, $\mathcal{M}_{P}(\lambda)$ can be expressed as the product of a global gain $k_{m}(P)$ with a spectrum curve $\mathcal{M}(\lambda)$, and (1) becomes:

$$
f(p)=k_{c} k_{m}(P) \int_{\lambda_{\min }}^{\lambda_{\max }} \mathcal{M}(\lambda) \mathcal{R}_{P}(\lambda) d \lambda
$$

Considering (1) and (2), the luminance $f$ can be modeled as a sum of three terms ${ }^{1}$ :

$$
f(p)=K_{d}(p) a(p) \cos \theta_{i}(p)+h(p)+K_{a}(p)
$$

\footnotetext{
${ }^{1}$ Note $P$ has been replaced by its projection into the sensor $p$.
} 
where the term $h$ refers to the specular reflection function (see 2.1) and includes a gain $K_{s}(p)$. Note also that $K_{d}(p)=k_{c} k_{m}(p) k_{d}(p), K_{s}(p)$ and $K_{a}(p)$ are the integration values respectively of $k_{c} k_{m}(p) k_{s}(P) \mathcal{M}(\lambda)$ and $k_{c} k_{m}(p) k_{a}(P) \mathcal{M}(\lambda)$ w.r.t the wavelengths.

The term noted $a(p)=\int_{\lambda_{\min }}^{\lambda_{\max }} \mathcal{M}(\lambda) \mathcal{R}_{P}^{b}(\lambda) d \lambda$ depends on the albedo $\mathcal{R}_{P}^{b}(\lambda)$, thus on the intrinsic property of the material.

\subsection{The luminance changes between two images of a sequence}

Two categories of illumination variations can occur between two successive frames, either they are due to a sole motion of the camera w.r.t $P$, or they are related to lighting changes (intensity change, or relative motion between the source and the object).

Motion of the camera. Some specular reflections can occur due to a simple motion of the camera with respect to the surface, leading to the displacement of $p$ towards $p^{\prime}$. The incident angle $\theta_{i}$ remains constant over time $\theta_{i}^{\prime}\left(p^{\prime}\right)=\theta_{i}(p)$ and if no lighting change occurs, $K_{d}$ and $K_{a}$ are also constant. Similarly, $a^{\prime}\left(p^{\prime}\right)=a(p)$ since it is an intrinsic property of the material. However, the specular component $h$ can vary since it depends on the viewing direction through the angle $\rho$. Thus, the luminance $f^{\prime}$ becomes

$$
f^{\prime}\left(p^{\prime}\right)=K_{d} a(p) \cos \theta_{i}(p)+h^{\prime}\left(p^{\prime}\right)+K_{a}
$$

By subtracting (4) from (5), it yields:

$$
f^{\prime}\left(p^{\prime}\right)=f(p)+\gamma(p) \text { with } \gamma(p)=h^{\prime}\left(p^{\prime}\right)-h(p)
$$

Lighting changes. Now, some lighting changes $\Delta K_{a}, \Delta K_{d}$ occur on $K_{a}$ and $K_{d}$ respectively. They stem from a shift of the camera gain, a variation 
of lighting intensity or shadows. Moreover, $\theta_{i}$ changes in $p$ according to a function $\Delta \theta_{i}$, and so the specular term $h^{\prime}\left(p^{\prime}\right)$. The luminance $f^{\prime}$ is then expressed as

$$
f^{\prime}\left(p^{\prime}\right)=K_{d}^{\prime}\left(p^{\prime}\right) a(p) \cos \theta_{i}^{\prime}\left(p^{\prime}\right)+h^{\prime}\left(p^{\prime}\right)+K_{a}^{\prime}
$$

with:

$$
\left\{\begin{array}{l}
K_{d}^{\prime}\left(p^{\prime}\right)=K_{d}(p)+\Delta K_{d}(p) \\
\theta_{i}^{\prime}\left(p^{\prime}\right)=\theta_{i}(p)+\Delta \theta_{i}(p) \\
K_{a}^{\prime}=K_{a}+\Delta K_{a} .
\end{array}\right.
$$

The specular term $h^{\prime}\left(p^{\prime}\right)$ includes the intensity change of the specular coefficient $K_{s}$. Therefore, by using (4) and (7), the relationship between two images of the same scene can be described by two different expressions:

1) First, it can be analogous to (6) by subtracting (4) from (7), where the function $\gamma$ is given by the following relationship:

$$
\begin{aligned}
\gamma(p)= & a(p)\left(K_{d}^{\prime}\left(p^{\prime}\right) \cos \left(\theta_{i}(p)+\Delta \theta_{i}(p)\right)-K_{d}(p) \cos \theta_{i}(p)\right)+ \\
& h^{\prime}\left(p^{\prime}\right)-h(p)+\Delta K_{a}
\end{aligned}
$$

Here, $\gamma(p)$ depends on $a(p)$ and thus on the albedo.

2) Second, it can be expressed by:

$$
f^{\prime}\left(p^{\prime}\right)=\alpha(p) f(p)+\beta(p)
$$

where:

$$
\left\{\begin{array}{l}
\alpha(p)=\frac{\left(K_{d}\left(p^{\prime}\right)+\Delta K_{d}\left(p^{\prime}\right)\right) \cos \left(\theta_{i}(p)+\Delta \theta_{i}(p)\right)}{K_{d}(p) \cos \theta_{i}(p)} \\
\beta(p)=-\left(h(p)+K_{a}\right) \alpha(p)+h^{\prime}\left(p^{\prime}\right)+K_{a}+\Delta K_{a} .
\end{array}\right.
$$

Note that neither $\alpha(p)$ nor $\beta(p)$ depend on $a(p)$, but only on the geometry. Due to the large number of parameters, among which the material properties 
(the roughness of the surface by the means of the specular terms), $\alpha(p)$ and $\beta(p)$ are not easy to compute and therefore have to be approximated.

\section{Local modeling of illumination changes in the image}

Generally speaking, the simplified photometric models rely on the modeling of luminance changes in local areas of the image. Therefore, they are available for image matching or feature point tracking when surfaces are assumed to be differentiable. Starting from (10) and (11), this section studies the assumptions on which these models are based. Let $\mathcal{W}$ be a window of interest centered in $p$ and $m$ a neighbor point belonging to $\mathcal{W}$.

\subsection{The luminance constancy}

From the radiance models of Section 2.1, the luminance constancy [9] is true only for Lambertian objects under constant lighting. In that case:

$$
f^{\prime}\left(m^{\prime}\right)=f(m) \text { for any } m \in \mathcal{W} .
$$

\subsection{The affine model}

The affine model assumes that the photometric variations are locally constant so that $\alpha(p)=\alpha$ and $\beta(p)=\beta$ in (10) leading to:

$$
f^{\prime}\left(m^{\prime}\right)=\alpha f(m)+\beta .
$$

Remark 1: the affine photometric model, given by (13) and the photometric normalization ([17] for example) are similar [30]. Let be $\mu_{f}$ and $\sigma_{f}$ (respectively $\mu_{f^{\prime}}$ and $\sigma_{f^{\prime}}$ ) the average and standard deviation of $f$ (respectively $f^{\prime}$ ) computed in $\mathcal{W}$. The normalization assumes the following 
relationship:

$$
\frac{f^{\prime}\left(m^{\prime}\right)-\mu_{f^{\prime}}}{\sigma_{f^{\prime}}}=\frac{f(m)-\mu_{f}}{\sigma_{f}}
$$

Therefore:

$$
\left\{\begin{array}{l}
\alpha=\frac{\sigma_{f^{\prime}}}{\sigma_{f}} \\
\beta=\mu_{f^{\prime}}-\frac{\sigma_{f^{\prime}} \mu_{f}}{\sigma_{f}}
\end{array}\right.
$$

Remark 2: The normalization (14) may introduce some noise for low $\sigma$ i.e for high luminance saturation or homogeneity in $\mathcal{W}$.

According to (11), the affine model (13) assumes that:

1) the surface is locally planar. Indeed, the incident angles $\theta_{i}$ and $\Delta \theta_{i}$ do not vary spatially in $\mathcal{W}$, i.e. when $\mathbf{n}$ is the same at each point of $\mathcal{W}$;

2) both objects and lighting are motionless ( $\theta_{i}$ constant);

3) the surface is Lambertian. Indeed, $h^{\prime}$ and $h$ are constant in $\mathcal{W}$. According to (2), this is true when both the $\rho$ and the roughness $r$ are constant in $\mathcal{W}$, i.e. when for all $m$ in $\mathcal{W}, h(m)=h^{\prime}\left(m^{\prime}\right)=0$ (no specular term).

\subsection{Some models suited for specular highlights occurrence and lighting changes}

The above assumptions are incorrect for non-planar surfaces, for which all the angles vary in $\mathcal{W}$. This section proposes two models which are less restrictive. The first one is available for small windows of interest.

\subsubsection{A photometric model for small areas}

As shown by Section 2, if only the camera moves, the luminance variations between two frames are properly described by (6), otherwise by (9).

According to $(2), \gamma$ is not constant in $\mathcal{W}$ since it depends on the angles and therefore on the normal $\mathbf{n}$ at each point of $\mathcal{W}$. It also depends on the $\gamma$ 
can be correctly approximated at first order on $\mathcal{W}$ by a $\mathcal{C}^{2}$ function ${ }^{2}$, noted $\gamma_{\text {mod }}$

which can be expanded in Taylor series, centered at a point $m$ with coordinates $(x, y)$, in the neighborhood of $p$ :

$$
\gamma(m) \simeq \gamma_{m o d}(p)+\left.\frac{\partial \gamma_{m o d}}{\partial x}\right|_{p}\left(x-x_{p}\right)+\left.\frac{\partial \gamma_{m o d}}{\partial y}\right|_{p}\left(y-y_{p}\right)
$$

Let be $\gamma_{1}=\left.\frac{\partial \gamma_{\text {mod }}}{\partial x}\right|_{p}, \gamma_{2}=\left.\frac{\partial \gamma_{\text {mod }}}{\partial y}\right|_{p}$ and $\gamma_{3}=\gamma_{\text {mod }}(p), \gamma=\left(\gamma_{1}, \gamma_{2}, \gamma_{3}\right)$ and $\mathbf{u}=\left(x-x_{p}, y-y_{p}, 1\right)$. By injecting (16) in (6) it yields:

$$
f^{\prime}\left(m^{\prime}\right)=f(m)+\gamma^{\top} \mathbf{u}
$$

This model is appropriate to model the photometric variations due to:

1) the motion of the camera, when there is no relative motion between the light source and the object. The surface projected onto $\mathcal{W}$ is not mandatory planar but its normal has to vary smoothly, and $K_{s}$ and $r$ as well (see (2)). In that context, the model (17) is well suited to compensate for specular highlights.

2) lighting changes, when the albedo and the normal $\mathbf{n}$ vary smoothly (see equation (9)). The approximation of $a(m)$ by a first order polynomial becomes more and more crude for large and textured surfaces.

\subsubsection{A photometric model for large areas}

According to (11), $\alpha$ depends on $\theta_{i}$, which varies on singular surfaces, especially on wide $\mathcal{W}$. Likewise, $\beta$ depends on the non-uniform specular highlights since they depend on $\mathbf{n}$ and $\rho$. However, it is reasonable to assume

\footnotetext{
${ }^{2}$ It implies that $\mathbf{n}$ varies smoothly in $\mathcal{W}$, i.e the surface is regular.
} 
that they are continuous and differentiable in each point $m$ when $\mathbf{n}, h$ and $r$ vary smoothly in $\mathcal{W}$. Then, $\alpha$ and $\beta$ can be expanded in Taylor series around $p$. By writing $\boldsymbol{\alpha}=\left(\left.\frac{\partial \alpha}{\partial x}\right|_{p},\left.\frac{\partial \alpha}{\partial y}\right|_{p}, \alpha(p)\right)$ and $\boldsymbol{\beta}=\left(\left.\frac{\partial \beta}{\partial x}\right|_{p},\left.\frac{\partial \beta}{\partial y}\right|_{p}, \beta(p)\right)$, it yields

$$
f^{\prime}\left(m^{\prime}\right)=\boldsymbol{\alpha}^{\top} \mathbf{u} f(m)+\boldsymbol{\beta}^{\top} \mathbf{u}
$$

This model assumes that the photometric changes can be non-uniform on a same $\mathcal{W}$. It is suited locally for both highlights and lighting changes, only when the surface is smooth. The validity of this model is studied more thoroughly in the next section.

\section{Study on the validity of the photometric model}

Consider a planar object ${ }^{3}$ viewed under one point light source at a known location. We compute the real photometric changes ( $\alpha$ and $\beta$ given by (11)) resulting from a change of the pose of the camera and the light source with regard to the object.

Second, we achieve a local approximation of these photometric functions by computing the second order Taylor series of $\alpha$ and $\beta$. The first order approximation of (18) is suited when at least its coefficients of second order are null or almost null. So, this study consists in finding these specific configurations ${ }^{4}$.

\footnotetext{
${ }^{3}$ Obviously, the photometric model will be less adequate for non-planar surfaces [31].

${ }^{4}$ To be comprehensive, the third and upper orders should be analyzed, but this fastidious analysis would have been detrimental to the clarity and concision of the paper.
} 


\subsection{Modeling of the scene geometry}

Consider a frame $\mathcal{F}_{c}$ linked to the camera. A point $P$ with coordinates $\left(X_{p}, Y_{p}, Z_{P}\right)$ is located at the center of a region of interest on the object. Let us also consider a point $M$, with coordinates $(X, Y, Z)$, located in the neighborhood of $P$. We assume that the surface in $P$ can be described as a $\mathcal{C}^{2}$ function leading to the following approximation of the depth in $M$, assumed to be valid in each point of $\mathcal{W}$ :

$$
Z=Z_{P}+D_{X}\left(X-X_{P}\right)+D_{Y}\left(Y-Y_{P}\right)
$$

where the first derivatives of the surface $D_{X}, D_{Y}$ describe the orientation between the tangent plane of the surface at $P$ and the CCD plane: $D_{X}=$ $\left.\frac{\partial Z}{\partial X}\right|_{P}$ and $D_{Y}=\left.\frac{\partial Z}{\partial Y}\right|_{P}$ The normal vector in $P$ is $\mathbf{n}=\left(\frac{\partial Z}{\partial X}, \frac{\partial Z}{\partial Y},-1\right)$.

Given $S=\left(S_{x}, S_{y}, S_{z}\right)$ (in the frame $\left.\mathcal{F}_{c}\right)$ the location of the light source, let $\mathbf{L}=\left(X-S_{x}, Y-S_{y}, Z\right)$ be the vector linking the lighting source $S$ to the point $M$. Then, $\cos \theta_{i}=\mathbf{L}$.n. By perspective projection and by using $Z$ given by (19), the real parameters $\alpha$ and $\beta$ given by (11) can be expressed with respect to the pixels coordinates $m$. Therefore some approximations and Taylor series expansions are achieved according to the acquisition configurations.

First of all, we study the validity of the approximation of $\alpha$ (section 3.3.2) by a first order polynomial. In order to simplify this study, we focus on small windows of interest $\mathcal{W}$ which are located near the optical axis of the camera. 


\subsection{Validity of the approximation of $\alpha$}

Let be $u=x-x_{P}$ and $v=y-y_{P}$ and consider the approximation of $\alpha$ (see equation (11)) at second order:

$$
\alpha(m)=\alpha_{1} u+\alpha_{2} v+\alpha_{3}+\alpha_{4} u^{2}+\alpha_{5} v^{2}+\alpha_{6} u v
$$

The lighting conditions for which $\left(\alpha_{4}, \alpha_{5}, \alpha_{6}\right)$ can be neglected are those for which the photometric model (18) better fits the illumination changes.

Only the direct light source is moved from its initial position $S$ with a small motion $\mathbf{d} \mathbf{S}=\left(d S_{X}, d S_{Y}, d S_{Z}\right)$. Indeed, a single motion of the light source impacts both $\alpha$ and $\beta$. Assuming a small $\mathbf{d S}$, the coefficients $\alpha_{i}$ can be expanded in Taylor series around $\mathbf{d S}$. Let us focus on the expression of $\alpha_{4}$ at first order, since the two other coefficients $\alpha_{5}=\alpha_{6}$ do not provide additional information. Three different configurations are considered.

1. The lighting vector $\mathbf{L}$ coincides with $\mathbf{n}: \mathbf{L}=\tau \mathbf{n}$ with $\tau$ constant:

$$
\alpha_{4}=\alpha_{5}=\alpha_{6}=0
$$

2. The light source is close to the camera $(S=O$ and $\mathbf{V}=\mathbf{L})$ :

$$
\alpha_{4}=-\frac{1}{Z_{P}}\left(2 d S_{Z}+2 D_{X} d S_{X}\right)
$$

3. The lighting source is close to the surface $\left(S=\left(X_{p}, Y_{p}, Z_{p}-\epsilon\right)\right.$ and Taylor expansion around $\epsilon=0)$.

$$
\alpha_{4}=-\frac{2 Z_{P}\left(Z_{P}+D_{X}^{2} \epsilon-Z_{P} D_{X}^{2}\right)}{\epsilon^{3}} d S_{Z}+\frac{2 Z_{P}\left(D_{X}(\epsilon-1)\right)}{\epsilon^{3}} d S_{X}
$$

Thus, our approximation of $\alpha$ is particularly well adapted when $\mathbf{L}$ coincides with $\mathbf{n}$ at the considered point (see (21)) and when the tangent plane of the 
surface at $P$ is parallel to the sensor plane i.e. when $\mathbf{V}$ coincides with $\mathbf{n}$ (indeed, $D_{X}=0$ in $(22)$ and $(23)$ ). The model is also well adapted when the depth of camera and light source are high (high $Z_{P}$ in (22)). When the lighting source is close to the surface, the model is correct when the camera is even closer to the surface (low $Z_{P}$ in (23)). Note also that a backward or forward motion of the light source influences the coefficient values, since $d S_{Z}$ appears in (22) and (23).

\subsection{Validity of the approximation of $\beta$}

In order to study the validity of the modeling of $\beta$ (expressed by (11)), it is necessary to consider the specular highlights and therefore the material properties. For this purpose, we use the Phong model (2). We assume a static object under constant lighting $\left(K_{a}\right.$ and $\left.K_{d}\right)$, so that $\alpha(m)=1$. Consequently, $\beta$ is equivalent to the function $\gamma$ in (6). Thus, we study the validity of the following expression: $\beta\left(m^{\prime}\right)=h^{\prime}(m)-h(m)$. $\beta$ is expanded in Taylor series at second order around $p$ :

$$
\beta(m)=\beta_{1} x+\beta_{2} y+\beta_{3}+\beta_{4} x^{2}+\beta_{5} y^{2}+\beta_{6} x y
$$

where the coefficients $\beta_{i}$ depend on the geometry parameters explained in section 4.1. Since $h$ reaches its maximum when $\rho$ is null, it is interesting to study the validity of the photometric models in this configuration, i.e $\mathbf{n}$ coincide with B (see Figure 1). As previously, we assume a small motion of the light source $\mathbf{d S}$ and achieve a Taylor series expansion of (24) around $\mathbf{d S}$. Some particular configurations of the scene geometry are studied in order to reach some simple conclusions. 
1. $\mathbf{L}=\mathbf{V}=\mathbf{n}$, therefore $D_{X}=D_{Y}=0$ and $\theta_{i}=0$. Since the expression of $\beta_{4}$ is far too complicated to deduce any useful information, let us focus on two particular configurations:

(a) The light source is close to the surface $\left(Z_{S}=Z_{P}\right)$, then:

$$
\beta_{4}=-r\left(\frac{1}{Z_{P}}\right) d S Z
$$

(b) The sensor is close to $P\left(Z_{P}\right.$ low, and Taylor series around $Z_{P}=$ $0)$, then:

$$
\beta_{4}=\beta_{5}=\beta_{6}=0
$$

2. Small orientation of the surface with regard to the sensor plane (small $D_{X}$ and $D_{Y}$ and Taylor series around $D_{X}=D_{Y}=0, \mathbf{n}=\mathbf{B}$ ).

(a) The light source is located near the surface $Z_{S}=Z_{P}$

$$
\eta_{4}=-\frac{r}{Z_{P}}\left(\frac{D_{X}}{4}(3 r+7) d S_{X}+\frac{D_{X}}{4}(r+1) d S_{Y}+d S_{Z}\right)
$$

(b) The sensor is close to the surface

$$
\beta_{4}=\beta_{5}=\beta_{6}=0
$$

According to (26) and (28), our photometric approximation is appropriate when the sensor is close to the surface. Otherwise, a forward (or a backward) motion $d S_{Z}$ of the light source w.r.t the surface induces variations of the $\beta_{i}$. A motion along the $Z$ axis has less impact when the sensor is far from the surface (large $Z_{P}$ ) and when the surface is rough (low $r$ in (25) or (27)).

In those conditions, the variations of the specular highlight draw up a plane on the window of interest $\mathcal{W}$, which can properly be compensated by the proposed illumination model. 


\subsection{Summary}

Table 1 provides an overview of the configurations for which the proposed photometric model is adapted $(+)$ or not $(-)$, or when the configuration has no influence $(=)$. Note that the affine photometric model (see section 3.2) relies on more restrictive assumptions, since $\alpha_{2}, \alpha_{3}, \beta_{2}, \beta_{3}$ have also to be null in (24) or (20). As said in section 3.2 page 10, the affine model is suited for Lambertian surfaces and is not adapted for a motion of a light source w.r.t to the surface.

The photometric models can be used for matching in order to improve some higher level procedures [21]. In this paper, we improve the robustness of KLT-like tracking.

\section{Feature points tracking algorithms}

Tracking features in a robust and accurate way along an image sequence is still an open problem, which explains the large variety of existing methods. The relative motion between the camera and the scene induces a geometric deformation described by the function $\delta$ which links $m^{\prime}$ to $m$ such that $\delta(m, \boldsymbol{\mu})=m^{\prime}$ forall $m \in \mathcal{W}$, according to a parameterization described by the vector $\boldsymbol{\mu}$. The feature point tracking consists in computing $\boldsymbol{\mu}$ according to a photometric model. We will show how to compute $\boldsymbol{\mu}$ for the photometric models given in Section 3.

\subsection{Commonly used tracking methods}

\subsubsection{The classical approach}

The classical KLT technique (for Kanade-Tomasi-Lucas tracker [7, 8]) assumes a Lambertian object, thus a perfect conservation of luminance at a 
Table 1: Overview of the results about the validity of the approximations of $\alpha$ and $\beta$ by a Taylor series expansion at first order. +: good approximation. -: bad approximation. =: there is no influence on the validity.

\begin{tabular}{|l|c|c|}
\hline Configuration & $\boldsymbol{\alpha}$ & $\boldsymbol{\beta}$ \\
\hline Lighting vector coincides with the normal & + & + \\
Viewing vector coincides with the normal & + & + \\
Rough surface & $=$ & + \\
Sensor close to the surface and lighting source & + & + \\
far from the surface & & \\
Motion of the lighting source along the optical & - & - \\
axis & & \\
\hline
\end{tabular}

point during the sequence (see $(12))$, so $f(m)=f^{\prime}(\delta(m, \boldsymbol{\mu}))$. However, as seen in Section 2, the luminance constancy assumption is not true. Besides, $\delta(m, \boldsymbol{\mu})$ is also an approximation. Thus, it is more judicious to minimize the following criterion:

$$
\epsilon_{1}(\boldsymbol{\mu})=\sum_{m \in \mathcal{W}}\left(f(m)-f^{\prime}(\delta(m, \boldsymbol{\mu}))\right)^{2}
$$

In order to obtain $\boldsymbol{\mu}$, we suppose that $\boldsymbol{\mu}=\widehat{\boldsymbol{\mu}}+\Delta \boldsymbol{\mu}$, where $\Delta \boldsymbol{\mu}$ expresses a small variation around an estimation $\widehat{\boldsymbol{\mu}}$ of $\boldsymbol{\mu}$. When $\widehat{\boldsymbol{\mu}}$ is unknown, it is initialized as $\widehat{\boldsymbol{\mu}}=0$. In those conditions, $f^{\prime}(\delta(m, \boldsymbol{\mu}))$ can be expanded in a first order Taylor series around $\widehat{\boldsymbol{\mu}}$ :

$$
f^{\prime}(\delta(m, \boldsymbol{\mu}))=f^{\prime}(\delta(m, \widehat{\boldsymbol{\mu}}))+\nabla f^{\prime \top}(\delta(m, \widehat{\boldsymbol{\mu}})) J_{\delta}^{\widehat{\boldsymbol{\mu}}} \Delta \boldsymbol{\mu}
$$

where $J_{\delta}^{\widehat{\mu}}$ is the Jacobian of $\delta$ according to $\boldsymbol{\mu}$, expressed in $\widehat{\boldsymbol{\mu}}$. Substituting (30) into (29) leads to a linear system in $\boldsymbol{\Delta} \boldsymbol{\mu}$, which can be solved iteratively: 


$$
\left(\sum_{m \in W} \mathbf{v}_{\mathbf{c}} \mathbf{v}_{\mathbf{c}}^{T}\right) \Delta \boldsymbol{\mu}=\sum_{m \in W}\left(f(m)-f^{\prime}(\delta(m, \widehat{\boldsymbol{\mu}}))\right) \mathbf{v}_{\mathbf{c}}
$$

with $\mathbf{v}_{\mathbf{c}}=\left(J_{\delta}^{\widehat{\boldsymbol{\mu}}}\right)^{\top} \nabla f^{\prime}(\delta(m, \widehat{\boldsymbol{\mu}}))$. When considering an affine motion model, $\mathbf{v}_{\mathbf{c}}$ is the vector defined by $\mathbf{v}_{\mathbf{c}}=\left({f^{\prime}}_{x}, f^{\prime}{ }_{y}, x{f^{\prime}}_{x}, x{f^{\prime}}_{y}, y f^{\prime}{ }_{x}, y{f^{\prime}}_{y}\right)^{\top}$, where ${f^{\prime}}_{x}$ and ${f^{\prime}}_{y}$ are the derivatives of $f^{\prime}$ with respect to $x$ and $y$ respectively.

\subsubsection{Tracking methods robust to affine photometric changes}

These approaches are based on the photometric model described in section 3.2. Therefore, the minimization function becomes:

$$
\epsilon_{2}(\boldsymbol{\mu}, \alpha, \beta)=\sum_{m \in W}\left(\alpha f(m)+\beta-f^{\prime}(\delta(m, \boldsymbol{\mu}))\right)^{2}
$$

where $\alpha$ and $\beta$ refer to the parameters of the affine model (13). There are two ways to obtain $\alpha$ and $\beta$, either by computing them outside the minimization loop (32) [17] or by computing them simultaneously with $\boldsymbol{\mu}$.

The photometric normalization. The tracking technique consists in computing $\boldsymbol{\mu}$ as in Section 5.1.1 since $\alpha$ and $\beta$ are constant. We have to solve:

$$
\left(\sum_{m \in W} \mathbf{v}_{c} \mathbf{v}_{c}^{\top}\right) \Delta \boldsymbol{\mu}=\sum_{m \in \mathcal{W}}\left(\alpha f(m)+\beta-f^{\prime}(\delta(m, \widehat{\boldsymbol{\mu}}))\right) \mathbf{v}_{c}
$$

where $\alpha$ and $\beta$ are computed using (15).

Estimation of $\alpha$ and $\beta$ : the Jin's technique. In [24], the authors propose to estimate the contrast $\alpha$ and intensity $\beta$ simultaneously with the motion model.

Let $\boldsymbol{\nu}$ be the vector of photometric variations $\boldsymbol{\nu}=(\alpha, \beta)$, and $\mathbf{d}$ the concatenation of $\boldsymbol{\mu}$ and $\boldsymbol{\nu}$. As previously, we assume a small variation $\Delta \mathbf{d}=$ 
$(\Delta \boldsymbol{\mu}, \Delta \boldsymbol{\nu})$ of $\mathbf{d}$ around its estimate $\widehat{\mathbf{d}}$ so that $\mathbf{d}=\widehat{\mathbf{d}}+\Delta \mathbf{d}$. Thus, by using (30) and introducing $\widehat{\boldsymbol{\nu}},(32)$ becomes

$$
\left(\sum_{m \in W} \mathbf{v}_{s} \mathbf{v}_{s}^{\top}\right) \Delta \mathbf{d}=\sum_{m \in \mathcal{W}}\left(\widehat{\alpha} f(m)+\widehat{\beta}-f^{\prime}(\delta(m, \widehat{\boldsymbol{\mu}}))\right) \mathbf{v}_{s}
$$

where $\mathbf{v}_{\mathbf{s}}=\left(-\mathbf{v}_{\mathbf{c}}, f(m), 1\right)^{\top}$.

Refer to Appendix A which compares the conditioning of the matrices used in the trackers detailed in the paper. The matrix $\sum_{m \in W} \mathbf{v}_{s} \mathbf{v}_{s}^{\top}$ is ill-conditioned and cannot be inverted easily, therefore a preconditioning, adapted to the image, is required.

The next section proposes two tracking procedures to account for nonuniform photometric changes.

\subsection{Proposed tracking procedures}

\subsubsection{A tracking method robust to specular highlights}

The first tracking method is based on the illumination model given by (17). The following criterion has to be minimized:

$$
\epsilon_{3}(\boldsymbol{\mu}, \gamma)=\sum_{m \in \mathcal{W}}\left(f(m)-f^{\prime}(\delta(m, \boldsymbol{\mu}))+\boldsymbol{\gamma}^{\top} \mathbf{u}\right)^{2}
$$

Let $\mathbf{d}=(\boldsymbol{\mu}, \boldsymbol{\gamma})$ and further assume a small motion $\Delta \mathbf{d}=(\Delta \boldsymbol{\mu}, \Delta \boldsymbol{\gamma})$ around an estimate $\widehat{\mathbf{d}}$ of $\mathbf{d}$. Similarly to the classical method of Section 5.1.1, $\Delta \mathbf{d}$ is computed by solving the following linear system:

$$
\left(\sum_{m \in \mathcal{W}} \mathbf{v}_{p} \mathbf{v}_{p}^{\top}\right) \Delta \mathbf{d}=\sum_{m \in \mathcal{W}}\left(f(m)-f^{\prime}(\delta(m, \widehat{\boldsymbol{\mu}}))+\widehat{\gamma}^{\top} \mathbf{u}\right) \mathbf{v}_{p}
$$

where $\mathbf{v}_{\mathbf{p}}=\left(-\mathbf{v}_{\mathbf{c}}, \mathbf{u}\right)^{\top}$. Unlike the previous tracker, the matrix $\left(\sum_{m \in \mathcal{W}} \mathbf{v}_{p} \mathbf{v}_{p}^{T}\right)$ is well conditioned (see Appendix A). 
According to the assumptions of the photometric model (17), this tracker is more appropriate to compensate for specular highlights. For small $\mathcal{W}$, it can also compensate for lighting changes when (9) can be approximated by a first order Taylor series expansion. For large windows, the next method is more appropriate.

\subsubsection{A tracking method robust to specular highlights and lighting changes}

Section 3.3.2 has detailed a comprehensive photometric model which compensates for spatial variations of specular highlights and lighting changes. The parameters $\boldsymbol{\mu}, \boldsymbol{\alpha}$ and $\boldsymbol{\beta}$ are computed by minimizing the following criterion

$$
\epsilon_{4}(\boldsymbol{\mu}, \boldsymbol{\alpha}, \boldsymbol{\beta})=\sum_{m \in W}\left(\boldsymbol{\alpha}^{\top} \mathbf{u} f(m)-f^{\prime}(\delta(m, \boldsymbol{\mu}))+\boldsymbol{\beta}^{\top} \mathbf{u}\right)^{2}
$$

The system can be linearized as in section 5.2.1, with $\mathbf{d}=(\boldsymbol{\mu}, \boldsymbol{\alpha}, \boldsymbol{\beta})$. Thus, the tracking consists in solving the following system:

$$
\left(\sum_{m \in \mathcal{W}} \mathbf{v}_{m} \mathbf{v}_{m}^{\top}\right) \Delta \mathbf{d}=\sum_{m \in \mathcal{W}}\left(\widehat{\boldsymbol{\alpha}}^{\top} \mathbf{u} f(m)-f^{\prime}(\delta(m, \widehat{\boldsymbol{\mu}}))+\widehat{\boldsymbol{\beta}}^{\top} \mathbf{u}\right) \mathbf{v}_{m}
$$

where $\mathbf{v}_{m}=\left(-\mathbf{v}_{c}, f(m) \mathbf{u}, \mathbf{u}\right)^{\top}$. Since the values of $\mathbf{v}_{m}$ are often dissimilar, the matrix $\sum_{m \in \mathcal{W}} \mathbf{v}_{m} \mathbf{v}_{m}^{\top}$ can be ill-conditioned (see Appendix A).

Moreover, the number of parameters is quite large. Indeed, by using an affine motion model, twelve parameters have to be computed. Obviously, the use of too small windows of interest may alter the accuracy of both photometric and motion estimations.

\section{Validation and experimental results}

This section compares the previous trackers through several sequences showing geometric and photometric changes simultaneously. First of all, we 
detail the experimental setup, the notations and the outlier rejection strategy. The first experiments are carried out on sequences for which a ground-truth is available in order to evaluate the methods as well as the outliers rejection procedure. Then, experiments are performed on real sequences, without ground-truth.

\subsection{Experimental setup and evaluation with ground-truth}

Tracking methods. The following notations are used:

C: classical tracking (section 5.1.1) for which $f^{\prime}(\delta(m, \boldsymbol{\mu}))=f(m)$

$\mathrm{N}$ : photometric normalization (section 5.1.2): $f^{\prime}(\delta(m, \boldsymbol{\mu}))=\lambda f(m)+\eta$

J: Jin et al. method (section 5.1.2): $f^{\prime}(\delta(m, \boldsymbol{\mu}))=\lambda f(m)+\eta$

$P_{3}$ : tracker with three parameters (section 5.2.1): $f^{\prime}(\delta(m, \boldsymbol{\mu}))=f(m)+\mathbf{u}^{\top} \boldsymbol{\gamma}$

$P_{6}$ : tracker with six parameters (section 5.2.2): $f^{\prime}(\delta(m, \boldsymbol{\mu}))=\boldsymbol{\alpha}^{\top} \mathbf{u} f(m)+\boldsymbol{\beta}^{\top} \mathbf{u}$

Size of the window of interest. Usually, the choice of the size $\mathcal{N} \times \mathcal{N}$ of the square window $\mathcal{W}$ is based on a trade-off between robustness to noise, computation times and reliability of the assumptions on which the tracker is based. Here, we consider some sizes ranging from $\mathcal{N}=9$ to $\mathcal{N}=35$.

Point rejection and comparison criteria. The points are selected in the first frame of the sequence by the Harris detector [5] and an affine motion model is computed between the first frame and the current frame.

The rejection process and its validation. The tracker has to constantly check whether the points are correctly tracked and are reliable ${ }^{5}$. To this end, most techniques analyze the residuals $[24,16]$. In our work, a point is rejected as soon as its residuals become higher than a threshold,

\footnotetext{
${ }^{5}$ They can be lost because of noise, disappearance or bad convergence.
} 
$\mathcal{S}_{\text {conv }}=\mathcal{N}^{2} E_{\text {ave }}^{2}$, where $E_{\text {ave }}$ is the tolerated luminance variation for each point in $\mathcal{W}$ between $f$ and its model. We choose $E_{\text {ave }}=15$. For each sequence, the following evaluation criteria are considered:

1) The robustness of the tracking, i.e. the number of points correctly tracked during the whole sequence.

2) The temporal evolution of the mean convergence residuals: $\left(1 / n_{k}\right) \sum_{i=1}^{n_{k}} \epsilon^{i}$, where $n_{k}$ is the number of points currently tracked by the considered method $k$. These two first criteria have to be considered jointly since the most satisfactory method is the one which tracks a large number of points with low residuals.

3) The location errors. When ground-truth is available, the mean location error is computed on all the points that are correctly tracked by the technique. Note that for a same location error, the best technique is the one which tracks the larger number of points.

Computation of the ground-truth. For planar surfaces, four blobs, easy to segment, are put on the object in order to compute the homography transform from the initial frame to the current one. For non planar surfaces, the pose is computed between the camera and the object, which is static (see [30] for the implementation details). In each case, the error is given by the euclidean distance between the theoretical points and the location provided by the tracker.

Sequences. In the two sequences used, there is no lighting change but the motion yields specular variations. Glossy paper has been chosen to cover the objects. The sequence Planar (Fig.2(a)) shows a planar surface of size $1 \times 1 \mathrm{~m}$. The camera is fixed at $Z_{P}=4 \mathrm{~m}$ and two light sources are located 
at $S_{z} \simeq 2 \mathrm{~m}$. The object is moved by an operator. The sequence Cylinder (Fig.2(b)) shows a cylinder of radius $7 \mathrm{~cm}$. The object and light sources are static, the camera is moving and $Z_{P} \simeq 1 \mathrm{~m}$ and $S_{z}>Z_{P}$.

\section{Results.}

1) Robustness. Table $2(a)$ and $2(b)$ page 26 show the percentage of correct points. For small windows, $P_{3}$ tracks the highest number of points. In contrast, for larger $\mathcal{W}, P_{6}$ is the most competitive method: on the planar surface it correctly tracks around twice the number of points. Despite its bad conditioning (see Appendix A), it outperforms $J$ or $N$ by better compensating the specular changes.

2) Location errors. Fig. 3 and 4 compare the mean location error computed on the correct points ${ }^{6}$. The location errors are low: around 1 pixel at the beginning of the sequence and less than 1 pixel when outliers points are rejected. The strategy for outliers rejection, based on the residuals, is therefore efficient to maintain a good accuracy of the tracking.

\subsection{Experiments on real sequences}

In the next sequences, the camera is moving and the scene is motionless: 1) Sequence Cylinder2 (150 images) (see Fig. 5a) shows a specular cylinder. The lighting conditions do not change but the motion of the camera causes some specular highlight variations on the object surface.

2) Sequences Planar object (Fig. 5b) and Marylin (Fig. 5c) show several textured objects consisting of several materials (glossy paper, ceramic, metal, cardboard, glass) under an ambient lighting (daylight and fluorescent lamps

\footnotetext{
${ }^{6}$ The method $C$ is not taken into account since too many points are lost.
} 
Table 2: Lab sequences. Percentage of points which have been correctly tracked during the sequence (occluded points or points going out of the image are not taken into account). $C$ : classical tracking. $N$ : tracking with photometric normalization. $J$ : Jin et al. method. $P_{3}$ : tracker with three photometric parameters. $P_{6}$ : tracker with six parameters.

(a) Planar surface.

\begin{tabular}{|l|lll|}
\hline $\mathcal{N}$ & 9 & 15 & 25 \\
\hline$C$ & 6 & 9 & 6 \\
$N$ & 29 & 32 & 32 \\
$J$ & 23 & 37 & 43 \\
$P_{3}$ & $\mathbf{3 4}$ & 34 & 37 \\
$P_{6}$ & 0 & $\mathbf{4 6}$ & $\mathbf{6 9}$ \\
\hline
\end{tabular}

(b) Cylinder.

\begin{tabular}{|l|llll|}
\hline $\mathcal{N}$ & 9 & 15 & 25 & 35 \\
\hline$C$ & 8 & 24 & 14 & 10 \\
$N$ & 2 & 30 & 36 & 40 \\
$J$ & 0 & 8 & 28 & 34 \\
$P_{3}$ & $\mathbf{1 0}$ & $\mathbf{4 0}$ & $\mathbf{7 0}$ & 62 \\
$P_{6}$ & 0 & 0 & 34 & $\mathbf{6 8}$ \\
\hline
\end{tabular}

located on the ceiling) and a direct light source. In the sequence Planar object, the intensity of the direct lighting varies strongly and periodically, with a period of about 20 frames, from a maximum value to a minimal one. The sequence Marylin is particularly complicated because of the large motion of the camera. In addition, some intensity variations of the light source are deliberately caused: around the iteration 135, the direct light is switched off, which induces some strong illumination changes.

3) Sequence Hill $7^{7}$ (Figure 5d) shows an outdoor scene acquired at different times of the day.

In sequences Cylinder2, Planar object and Marylin, $Z_{P} \simeq S_{z}$, which is

\footnotetext{
${ }^{7}$ This sequence can be found in the image database CMU/VASC : http://vasc.ri.cmu.edu/idb/html/motion/index.html
} 
not a favorable case for any photometric models (see section 4 and Table 1). In the sequence Hill $S_{z}>>Z_{P}$, which is a favorable configuration. Each sequence is played forward and backward, in order to check the good behavior of the tracking, i.e the symmetry of residuals curve.

Results. Table 3 reports the percentage of correct points w.r.t $\mathcal{N}$, for each tracking technique and each sequence. $P_{3}$ tracks the largest number of points for $\mathcal{N} \leq 15$ (up to $\mathcal{N}=25$ for Cylinder2). $P_{6}$ does not converge ${ }^{8}$ for small $\mathcal{N}$ but performs well for $\mathcal{N} \geq 25$.

As noticed in section 6.1, the behavior of the mean residuals has also to be considered. As can be seen on Fig.6 to 9, the residuals evolve in a similar way as the illumination changes. For instance on Fig. 7, they vary periodically with the same frequency as the lighting. In most sequences, for $\mathcal{N}=9, P_{3}$ provides the lowest residuals, although these values are computed from a larger number of points than $J$ and $N$ (see Table 3 ) especially in Marylin. $P_{3}$ offers almost similar residuals as $J$ and $N$ in Planar surface and Hill but it performs better when only specular highlights are considered (see Fig. 6b). In contrast, its residuals are higer when lighting changes occur (see Figures $7 b$ and $8 c$ ) except for the Hill sequence (see Figure $9 c$ ), which offers a favorable configuration for the photometric models (see section 3.3.1). (see the residuals on the Fig. $7 c, 8 c, 9 c) P_{6}$ always yields the lowest residuals and tracks the largest number of points. As an example of the relevance of the photometric correction, Fig. 10 (a) shows the temporal evolution of the luminance of a window $\mathcal{W}$ tracked in the sequence of Fig. 7 and Fig. 10 (b)

\footnotetext{
${ }^{8}$ The number of unknown parameters is too large to be properly estimated by the low number of pixels in $\mathcal{W}$.
} 
shows the same $\mathcal{W}$ after proper photometric correction by $P_{6}$. The evolutions of the spatial photometric corrections $\boldsymbol{\alpha}^{\top} \mathbf{u}$ and $\boldsymbol{\beta}^{\top} \mathbf{u}$ in $\mathcal{W}$ are displayed on Fig. 10 (c) and Fig. 10 (d) respectively.

Computation times. Consider a point correctly tracked by each method. The computation times are reported in Table 4 , for $\mathcal{N}=9,15$ and 35. Obviously $N$ and $P_{6}$ are the most time-consuming techniques, either because of the computation of the photometric normalization or because of the high number of photometric parameters. In addition, they might require a larger number of iterations to converge. For $\mathcal{N}=9$ and $15, C, J$ and $P_{3}$ have similar computation times ${ }^{9}$.

\subsection{Discussion}

On small windows of interest, $J, N$ and $P_{6}$ perform bad compared to $C$ and $P_{3}$. This can be partly explained by a sensitivity to image noise: when a pixel is noisy in $\mathcal{W}$, the values of $\mu_{f}, \sigma_{f}, \mu_{g}, \sigma_{g}$ (see (15)), and $\alpha$ also become noisy since they depend on the luminance. For $J$ and $P_{6}, \alpha$ is multiplied by $f$, consequently an error on $\alpha$ can have a huge influence and the minimization can lead to an incorrect value of $\boldsymbol{\mu}$.

For small $\mathcal{W}, P_{3}$ tracks a larger number of points than $N$ and $J$ by correctly compensating for the specular highlights and lighting changes. Its performance is reduced on large $\mathcal{W}$ under lighting changes since the model has to approximate somehow the albedo of the object by a first order polynomial on $\mathcal{W}$, which is a strong assumption when reflectance varies drastically.

Note also that, even if $P_{3}$ requires the computation of an additional pa-

\footnotetext{
${ }^{9}$ However, note that the KLT trackers can be accelerated on GPU units as in [32].
} 
Table 3: Percentage of the points that have been tracked up to the end of the sequence with respect to the points which were initially selected, with regard to $\mathcal{N}$.

(a) Cylinder2 (137 points selected) $\quad$ (b) Planar object (58 points are se-

\begin{tabular}{|l|llll|}
\hline $\mathcal{N}$ & 9 & 15 & 25 & 35 \\
\hline$C$ & 86.5 & 80.2 & 71.4 & 60.5 \\
$N$ & 40.6 & 69.5 & 83.3 & 71.4 \\
$J$ & 76.7 & 85.5 & 85.7 & 73.9 \\
$P_{3}$ & $\mathbf{9 6 . 2}$ & $\mathbf{9 3 . 1}$ & $\mathbf{8 7 . 3}$ & 77.3 \\
$P_{6}$ & - & 64.8 & 81.7 & $\mathbf{8 8 . 2}$ \\
\hline
\end{tabular}
lected)

(d) Hill (156 points are selected)

\begin{tabular}{|l|llll|}
\hline $\mathcal{N}$ & 9 & 15 & 25 & 35 \\
\hline$C$ & 63.8 & 36.2 & 8.6 & 6.9 \\
$N$ & 77.6 & 91.4 & 96.6 & 93.1 \\
$J$ & 67.2 & 51 & 87.9 & 89.7 \\
$P_{3}$ & $\mathbf{1 0 0}$ & $\mathbf{1 0 0}$ & 96.6 & 96.6 \\
$P_{6}$ & 48.3 & 94.8 & $\mathbf{1 0 0}$ & $\mathbf{1 0 0}$ \\
\hline
\end{tabular}

\begin{tabular}{|l|llll|}
\hline $\mathcal{N}$ & 9 & 15 & 25 & 35 \\
\hline$C$ & 49.6 & 20 & 11.2 & 9.6 \\
$N$ & 45.9 & 63.7 & 73.7 & 75 \\
$J$ & 55.6 & 70.4 & 85.6 & 93.3 \\
$P_{3}$ & $\mathbf{7 4 . 8}$ & 75.6 & 86.4 & 95.2 \\
$P_{6}$ & - & $\mathbf{7 7 . 8}$ & $\mathbf{8 9}$ & $\mathbf{9 7 . 1}$ \\
\hline
\end{tabular}

(e) Marylin (56 points are selected)

\begin{tabular}{|l|llll|}
\hline $\mathcal{N}$ & 9 & 15 & 25 & 35 \\
\hline$C$ & 0 & 0 & 0 & 0 \\
$N$ & 0 & 21.4 & 17.9 & 17.9 \\
$J$ & 0 & 7.2 & 10.7 & 17.9 \\
$P_{3}$ & $\mathbf{4 6 . 4}$ & 14.3 & 7.2 & 3.6 \\
$P_{6}$ & - & 14.3 & $\mathbf{4 2 . 9}$ & $\mathbf{3 9 . 3}$ \\
\hline
\end{tabular}

rameter w.r.t $J$ and consequently the inversion of a wider matrix, the computation times of these techniques are similar, due to a better convergence of $P_{3}$.

$P_{6}$ is the most accurate tracker for large $\mathcal{W}$, because the use of a comprehensive photometric model improves the estimation of the motion model during the sequence. 
Table 4: Computation times (in ms) of the tracking of one point in the sequence Cylinder2, and planar object for $\mathcal{N}=9,15$ et 35 . (- = no convergence)

Specular highlights

\begin{tabular}{|c|ccc|}
\hline Method & $\mathcal{N}=9$ & $\mathcal{N}=15$ & $\mathcal{N}=35$ \\
\hline$C$ & 1.3 & 2.7 & 21 \\
$N$ & 4.6 & 6.8 & 31.2 \\
$J$ & 1.7 & 3.1 & 21.7 \\
$P_{3}$ & 1.4 & 3.2 & 12.3 \\
$P_{6}$ & - & 8.5 & 25 \\
\hline
\end{tabular}

Lighting changes

\begin{tabular}{|c|ccc|}
\hline Method & $\mathcal{N}=9$ & $\mathcal{N}=15$ & $\mathcal{N}=35$ \\
\hline$C$ & 1.3 & 2.9 & 11.4 \\
$N$ & 4.3 & 3.5 & 14.1 \\
$J$ & 1.6 & 3.2 & 11.5 \\
$P_{3}$ & 2 & 3.5 & 13.8 \\
$P_{6}$ & - & 5.9 & 18.4 \\
\hline
\end{tabular}

\section{Conclusions}

Through the analysis of specular reflection models, this paper has explained the assumptions on which the most widely used photometric models are implicitly based. Then, it has proposed some local photometric models which rely on a precise analysis of the reflection, and on the assumption that illumination changes vary smoothly in local areas of the image.

The proposed photometric models can be useful in many computer vision applications where lighting is not perfectly controlled, especially in outdoor experiments or for mobile cameras. This paper incorporated the models into two KLT feature point tracking procedures.

By compensating for the spatial variations of illumination changes, the proposed methods have proved to be more robust than the existing ones. The first tracker is well adapted for small windows of interest, while the 
second one is applicable for larger windows of interest. Experimental results on several images sequences have shown good convergence and accuracy for these procedures. Future work will investigate the combination of the two photometric models depending on the characteristics of the point to track and depending on the local characteristics of illumination changes that are computed during the sequence.

\section{References}

[1] A. Comport, E. Malis, P. Rives, Accurate quadrifocal tracking for robust 3D visual odometry, in: IEEE Int. Conf. on Robotics and Automation ICRA'07, 2007, pp. 40-45.

[2] F. Espiau, E. Malis, P. Rives, Robust features tracking for robotic applications: towards 2D 1/2 visual servoing with natural, in: IEEE Int. Conf. on Robotics and Automation, ICRA'02, Washington, USA, 2002.

[3] D. Ziou, S. Tabbone, Edge detection techniques - An overview, Int. Journ. of Pattern Recognition and Image Analysis 8 (1998) 537-559.

[4] M. Pressigout, E. Marchand, Real-time 3D model-based tracking: Combining edge and texture information, in: IEEE Int. Conf on Robotics and Automation, ICRA'06, Orlando, Florida, 2006.

[5] C. Harris, M. Stephens, A combined corner and edge detector, in: 4th Alvey Vision Conference, 1988, pp. 147-151.

[6] S. Bouchafa, B. Zavidovique, Efficient cumulative matching for image registration, Image and Vision Computing 24 (1) (2006) 70-79. 
[7] B. Lucas, T. Kanade, An iterative image registration technique, in: IJCAI'81, Vancouver, British Columbia, 1981, pp. 674-679.

[8] C. Tomasi, T. Kanade, Detection and tracking of point features, Technical report CMU-CS-91-132, Carnegie Mellon University (April 1991).

[9] K. Horn, B. G. Schunck, Determinig optical flow, Artificial Intelligence 7 (1981) 185-203.

[10] J. Shi, C. Tomasi, Good features to track, in: IEEE Int. Conf. on Computer Vision and Pattern Recognition, CVPR'94, Seattle, Washington, USA, 1994, pp. 593-600.

[11] J. Odobez, P. Bouthemy, Robust multiresolution estimation of parametric motion models, Int. Journ. of Visual Communication and Image Representation 6 (4) (1995) 348-365.

[12] J. Buenaposada, L. Baumela, Real-time tracking and estimation of plane pose, in: IEEE Int. Conf. on Pattern Recognition, Vol. 2, Quebec, Canada, 2002, pp. 697-700.

[13] C. Collewet, A. Alhaj, F. Chaumette, Model-free visual servoing on complex images based on 3D reconstruction, in: IEEE Int. Conf. on Robotics and Automation ICRA'04, New Orleans, USA, 2004.

[14] P. Tissainayagam, D. Suter, Assessing the performance of corner detectors for point feature tracking applications, Image and Vision Processing 22 (6) (2004) 663-679. 
[15] S. Benhimane, E. Malis, Homography-based 2D visual tracking and servoing, The Int. Journ. of Robotics Research 26 (7) (2007) 661-676.

[16] G. D. Hager, P. N. Belhumeur, Efficient region tracking with parametric models of geometry and illumination, IEEE Trans. on Pattern Analysis and Machine Intelligence 20 (10) (1998) 1025-1039.

[17] T. Tommasini, A. Fusiello, E. Trucco, V. Roberto, Improving feature tracking with robust statistics, Pattern Analysis \& Applications 2 (1999) $312-320$.

[18] S. Negahdaripour, Revised definition of optical flow: integration of radiometric and geometric cues for dynamic scene analysis, IEEE Transactions on Pattern Analysis and Machine Intelligence 20 (9) (1998) 961 $-979$.

[19] H. Hausseker, D. Fleet, Computing optical flow with physical models of brightness variation, in: IEEE Int. Conf. on Computer Vision and Pattern Recognition, Vol. 2, Hilton Head, 2000, pp. 760-767.

[20] S.-H. Lai, Computation of optical flow under non-uniform brightness variations, Pattern Recognition Letters 25 (8) (2004) 885 - 892.

[21] M. Black, D. Fleet, Y.Yacoob, Robustly estimating changes in image appearance, Computer Vision and Image Understanding 78 (2000) 8 31.

[22] A. S. Georghiades, P. Belhumeur, D. J. Kriegman, From few to many: generative models for recognition under variable pose and illumination, 
IEEE Trans. on Pattern Analysis and Machine Intelligence 23 (8) (2001) $643-660$.

[23] P. Gros, Color illumination models for image matching and indexing, in: IEEE Int. Conf. on Pattern Recognition ICPR'00, Barcelona, Spain, 2000.

[24] S. Soatto, H. Jin, P. Favaro, Real-time feature tracking and outlier rejection with changes in illumination, in: IEEE Int. Conf. on Computer Vision, Vancouver, Canada, 2001, pp. 684-689.

[25] G. Silveira, E. Malis, Real-time visual tracking under arbitrary illumination changes, Minneapolis, USA, 2007.

[26] B.-T. Phong, Illumination for computer generated images, Communications of the ACM 18 (6) (1975) 311-317.

[27] K. Torrance, E. Sparrow, Theory for off-specular reflection from roughened surfaces, Journ. of the Optical Society of America 57(9).

[28] J. Lambert, Photometria sive de mensura de gratibus luminis, colorum et ombrae, Ebrhard Klett, Augsburg, 1760.

[29] J. F. Blinn, Models of light reflection for computer synthesized pictures, in: Proc. 4th annual Conference on Computer Graphics and Interactive Techniques SIGGRAPH '77, 1977, p. 192.

[30] M. Gouiffès, C. Collewet, C. Fernandez-Maloigne, A. Trémeau, Modeling illumination changes for matching correspondances, its validity 
and its application to robust tracking, available on http://hal.archivesouvertes.fr/, Tech. Rep. hal-00430799, IEF, Univ. Paris Sud 11, CNRS UMR 8622 (2007).

[31] M. Gouiffès, C. Collewet, C. Fernandez-Maloigne, A. Trémeau, A photometric model for specular highlights and lighting changes. Application to feature points tracking., in: IEEE Int. Conf. on Image Processing ICIP'06, 2006.

[32] J.-S. Kim, M. Hwangbo, T. Kanade, Realtime affine-photometric klt feature tracker on gpu in cuda framework, in: Workshop on Embedded Computer Vision (ECV), 2009.

\section{Appendix A. Conditioning}

The conditioning of the trackers detailed in that report can be compared. Indeed, each linear equation system involved in the tracking procedure (see equations (31), (33), (34), (36) and (38)) can be written as $\mathbf{A x}=\mathbf{y}$ and more precisely

$$
\left(\begin{array}{cc}
\mathrm{A}_{11} & \mathrm{~A}_{12} \\
\mathrm{~A}_{12}^{\top} & \mathrm{A}_{22}
\end{array}\right)\left(\begin{array}{l}
\mathrm{x}_{1} \\
\mathrm{x}_{2}
\end{array}\right)=\left(\begin{array}{l}
\mathrm{y}_{1} \\
\mathrm{y}_{2}
\end{array}\right)
$$

Consequently, the inversion of $\mathbf{A}$ is given by

$$
\mathbf{A}^{-1}=\left(\begin{array}{cc}
\mathbf{I}_{3} & 0 \\
-\mathbf{A}_{22}{ }^{-1} \mathbf{A}_{12}{ }^{\top} & \mathbf{I}_{3}
\end{array}\right)\left(\begin{array}{cc}
\nabla^{-1} & 0 \\
0 & \mathbf{A}_{22}{ }^{-1}
\end{array}\right)\left(\begin{array}{cc}
\mathbf{I}_{3} & -\mathbf{A}_{12} \mathbf{A}_{22}{ }^{-1} \\
0 & \mathbf{I}_{3}
\end{array}\right)
$$

where $\boldsymbol{\nabla}$ is the Schur complement $\boldsymbol{\nabla}=\mathbf{A}_{\mathbf{1 1}}-\mathbf{A}_{\mathbf{1 2}} \mathbf{A}_{\mathbf{2 2}}{ }^{-1} \mathbf{A}_{\mathbf{1 2}}{ }^{\top}$. The inversion of $\boldsymbol{\nabla}$ can be achieved in the following way:

$$
\boldsymbol{\nabla}^{-1}=\mathbf{A}_{11}{ }^{-1}+\mathbf{A}_{11}{ }^{-1} \mathbf{A}_{12}\left(\mathbf{A}_{22}-\mathbf{A}_{12}{ }^{\top} \mathbf{A}_{11}{ }^{-1} \mathbf{A}_{12}\right)^{-1} \mathbf{A}_{12}{ }^{\top} \mathbf{A}_{11}{ }^{-1}
$$


Since $\left(\mathbf{A}_{\mathbf{1 2}} \mathbf{A}_{\mathbf{2 2}}{ }^{-1}\right)^{\top}=\mathbf{A}_{\mathbf{2 2}}{ }^{-1^{\top}} \mathbf{A}_{\mathbf{1 2}}{ }^{\top}$ we can introduce :

$$
\mathbf{M}=\left(\begin{array}{cc}
\mathbf{I}_{3} & -\mathbf{A}_{12} \mathbf{A}_{22}{ }^{-1} \\
0 & \mathbf{I}_{3}
\end{array}\right)
$$

so that

$$
\mathbf{A}^{-1}=\mathbf{M}^{\top}\left(\begin{array}{cc}
\boldsymbol{\nabla}^{-1} & 0 \\
0 & \mathbf{A}_{\mathbf{2 2}}{ }^{-1}
\end{array}\right) \mathbf{M}
$$

Consequently, the inversion of $\mathbf{A}$ succeeds if $\boldsymbol{\nabla}$ and $\mathbf{A}_{\mathbf{2 2}}$ are well-conditioned and can be correctly inverted. $\boldsymbol{\nabla}$ is well conditioned when $\mathbf{A}_{\mathbf{1 1}}$ is wellconditioned (see (A.3)).

In the tracking techniques, for each approach the matrix $\mathbf{A}_{\mathbf{1 1}}$ is the same. Therefore, the comparison of the conditioning of the method only depends on the conditioning of $\mathbf{A}_{\mathbf{2 2}}$. The matrices associated to the methods which approximate the photometric parameters $\mathbf{A}_{\mathbf{2 2}}{ }^{J}$ (for the Jin's technique), $\mathbf{A}_{\mathbf{2 2}}{ }^{P_{3}}$ and $\mathbf{A}_{\mathbf{2 2}}{ }^{P_{6}}$ are written as:

$$
\begin{array}{ll}
J & \mathbf{A}_{\mathbf{2 2}}{ }^{J}=\sum_{m}(f(m), 1)(f(m), 1)^{\top} \\
P_{3}: & \mathbf{A}_{\mathbf{2 2}}{ }^{P_{3}}=\sum_{m} \mathbf{u u}^{\top} \\
P_{6}: & \mathbf{A}_{\mathbf{2 2}}{ }^{P_{6}}=\sum_{m}(\mathbf{u} f(m), \mathbf{u})(\mathbf{u} f(m), \mathbf{u})^{\top}
\end{array}
$$

The matrix $\mathbf{A}_{\mathbf{2 2}}{ }^{P_{3}}$ is the best well-conditioned. On the contrary, the matrix $\mathbf{A}_{\mathbf{2 2}}{ }^{J}$ and $\mathbf{A}_{\mathbf{2 2}}{ }^{P_{6}}$ are ill-conditioned and their terms depend on the image through $f(m)$. 


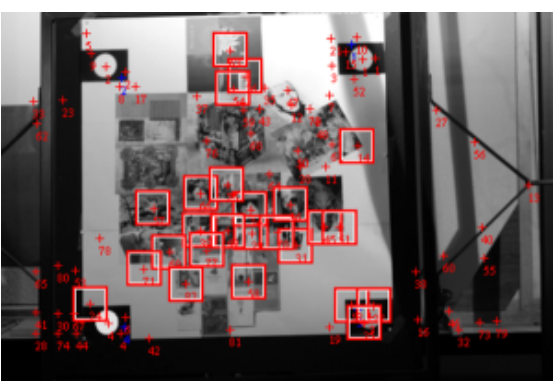

(a)

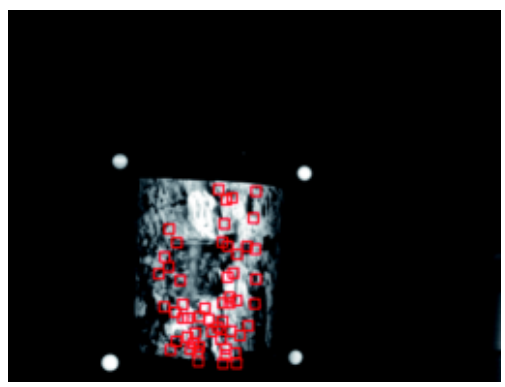

(b)

Figure 2: Sequences used to evaluate the accuracy of the tracking procedures. (a) PlanAR Surface. (b) Cylinder.
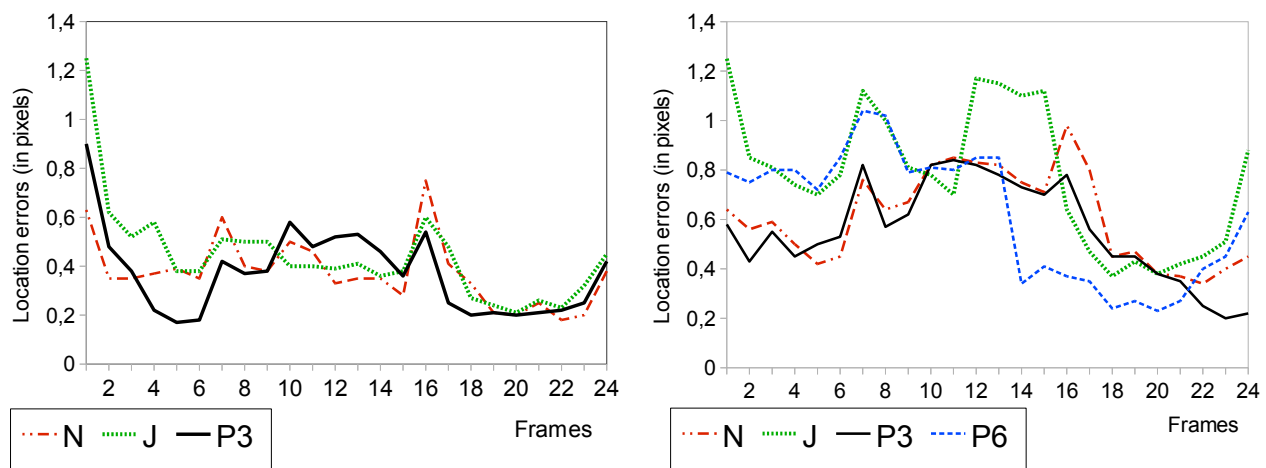

Figure 3: Planar surface. Convergence residuals and location errors for $\mathcal{N}=9$ (left) and $\mathcal{N}=25$ (right).
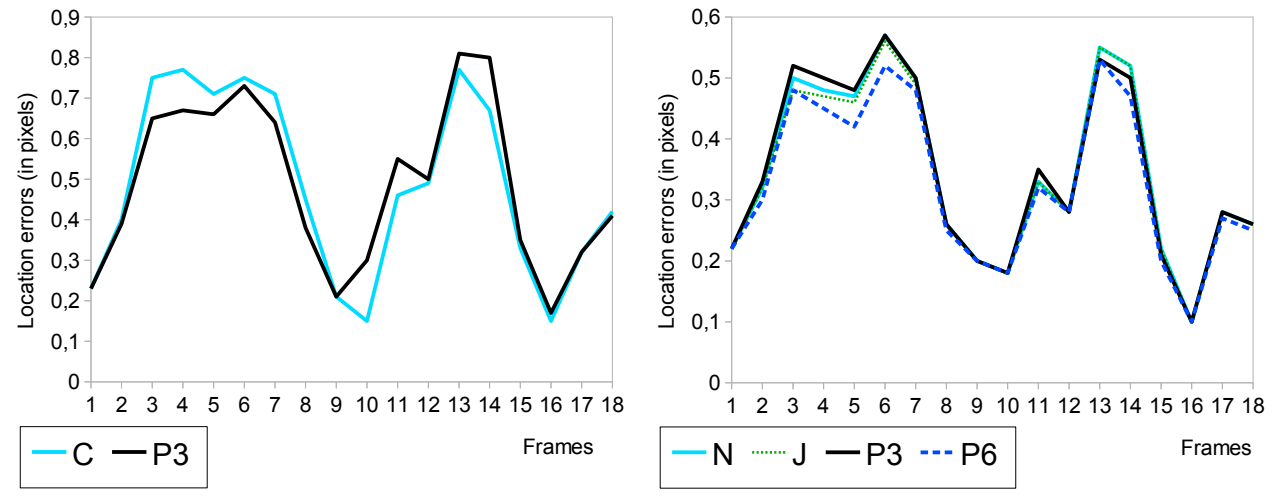

Figure 4: Cylinder. Convergence residuals and location errors for $\mathcal{N}=9$ (left) and $\mathcal{N}=25$ (right). 
(a)
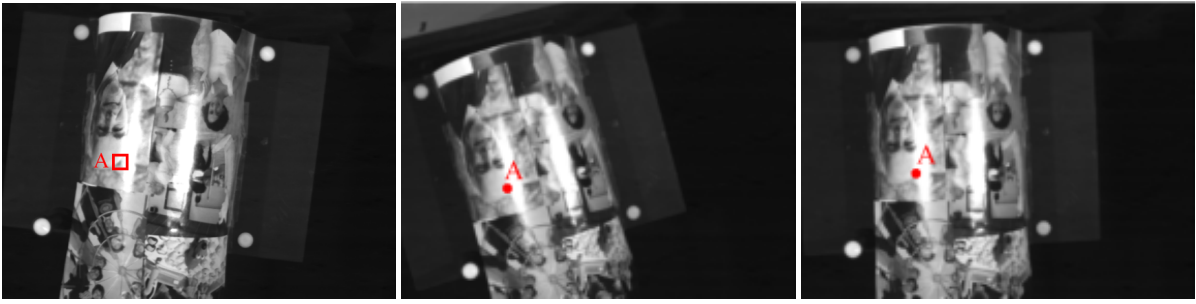

$1^{\text {rst }}$ frame

$110^{\text {th }}$ frame

$150^{\text {th }}$ frame

(b)
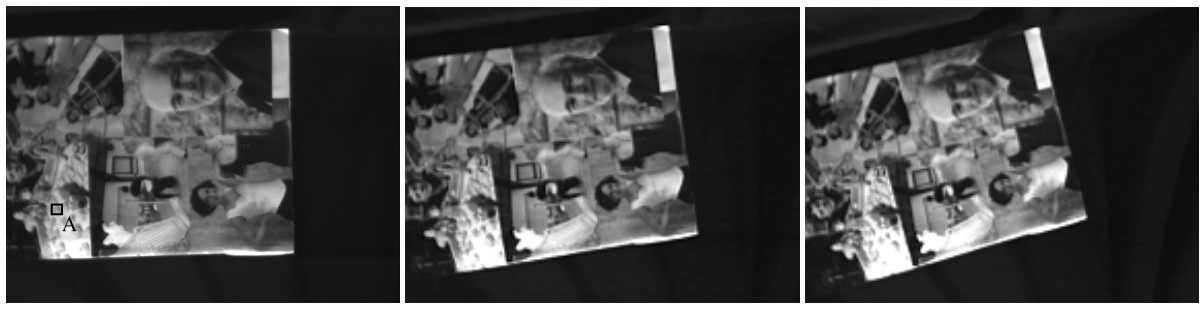

$1^{r s t}$ frame

$75^{\text {th }}$ frame

$150^{\text {th }}$ frame

(c)
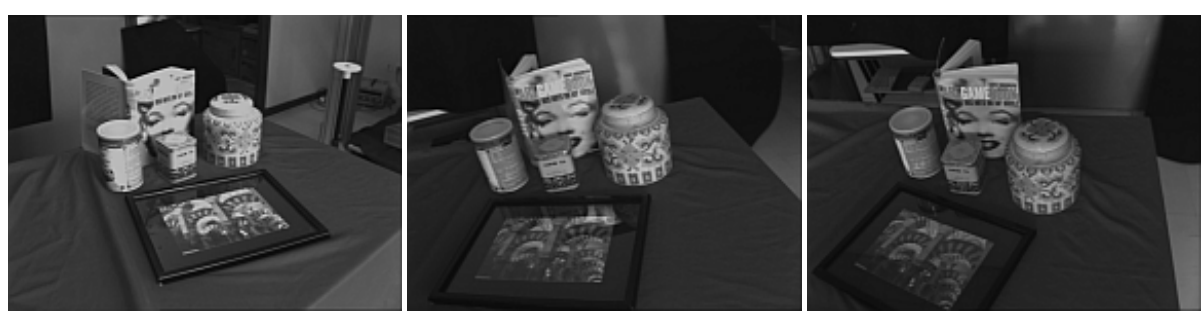

$1^{r s t}$ frame

$145^{\text {th }}$ frame

$299^{\text {th }}$ frame

(d)
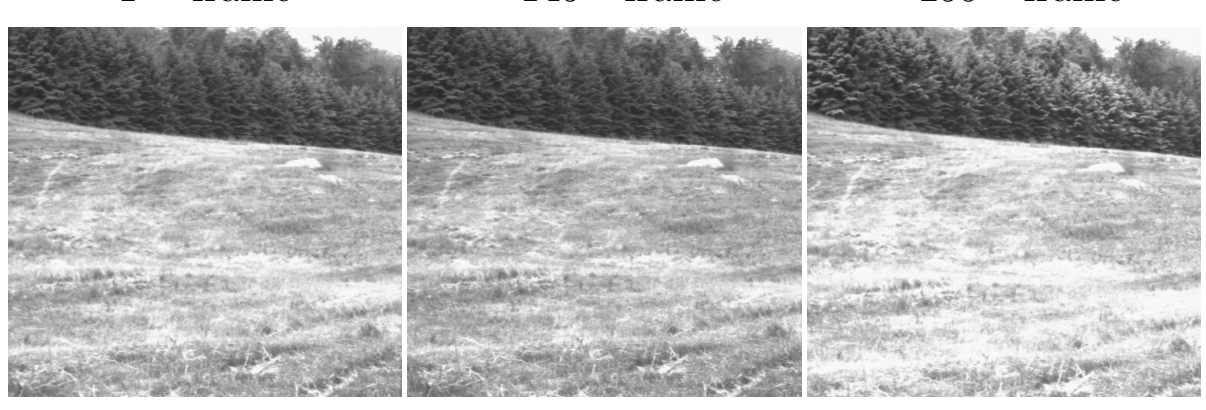

$2^{\text {nd }}$ frame

$9^{\text {th }}$ frame

$13^{\text {th }}$ frame

Figure 5: Images sequences. (a) Cylinder2. (b) Planar object. (c) Marylin. (d) Hill. 


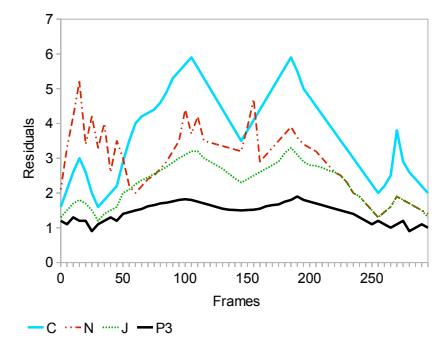

(a) $\mathcal{N}=9$

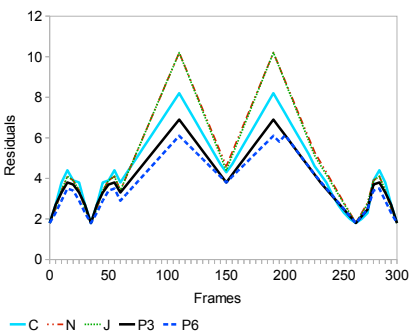

(b) $\mathcal{N}=35$

Figure 6: Sequence Cylinder2. 37 points have been selected. Average of the residuals with regard the frame number, with a window size (a) $\mathcal{N}=9$ (b) $\mathcal{N}=35$.

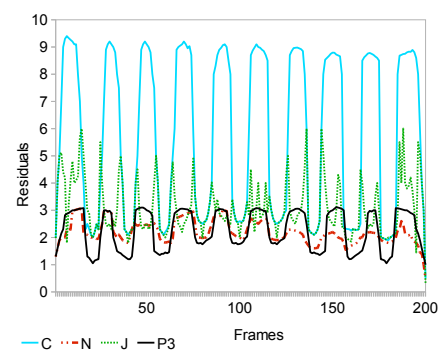

(a) $\mathcal{N}=9$

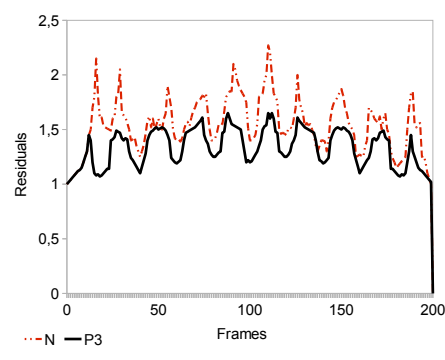

(b) $\mathcal{N}=9$

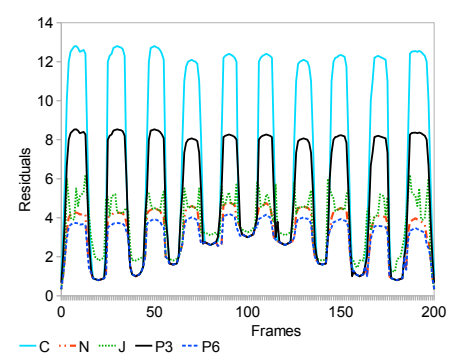

(c) $\mathcal{N}=35$

Figure 7: Sequence Planar object. (a) Average residuals obtained with $\mathcal{N}=9$. (b) Residuals obtained with $\mathcal{N}=9$ on the points which are tracked simultaneously by $N$ and $P_{3}$. (c) Average residuals obtained with $\mathcal{N}=35$.

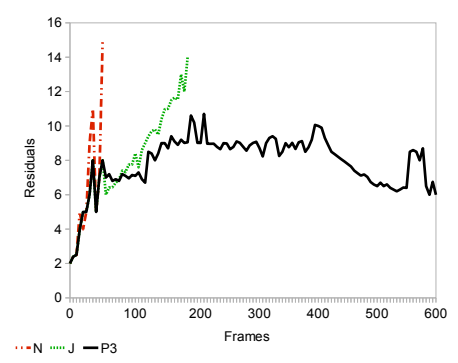

(a) $\mathcal{N}=9$

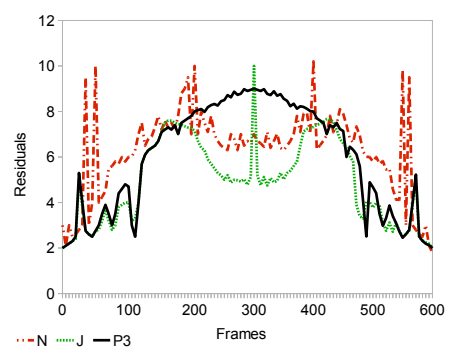

(b) $\mathcal{N}=15$

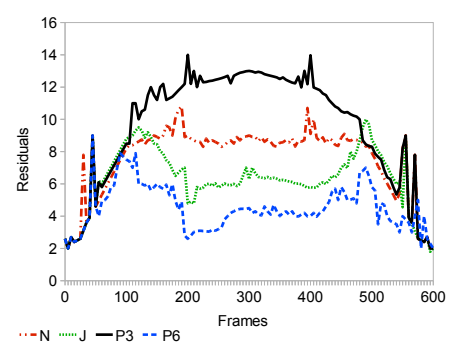

(c) $\mathcal{N}=35$

Figure 8: Sequence Marilyn. Average residưls obtained with $(a): \mathcal{N}=9,(\mathrm{~b}): \mathcal{N}=15$ and (c): $\mathcal{N}=35$. 


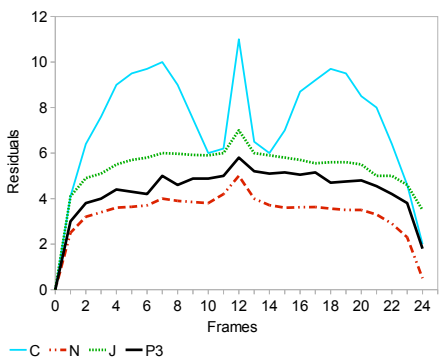

(a) $\mathcal{N}=9$

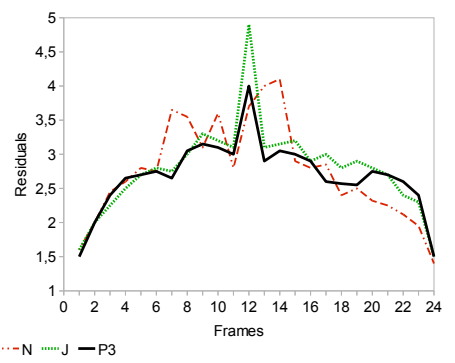

(b) $\mathcal{N}=15$

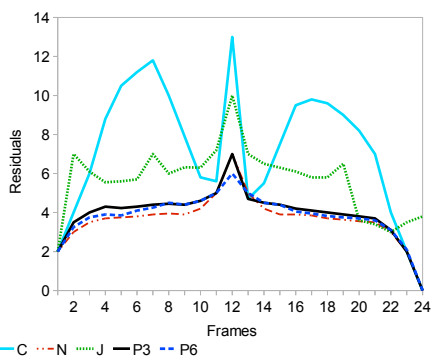

(c) $\mathcal{N}=35$

Figure 9: Sequence Hill. (a) Average residuals obtained with $\mathcal{N}=9$. (b) Residuals obtained with $\mathcal{N}=9$ on the points which are tracked simultaneously by $N$ and $P_{3}$. (c) Average residuals obtained with $\mathcal{N}=35$.

(1)

(2)

$\mathrm{k} \quad 10 \quad 20$
(3)

(4)

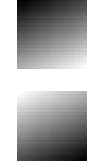

$\mathrm{k} \quad 10$

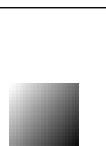

1

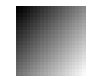

20
(1)

$(2)$

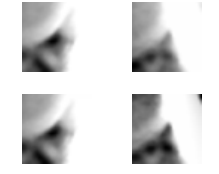

(3)

(4)
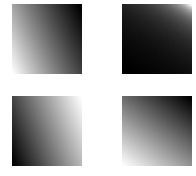

$20 \quad 100 \quad \mathrm{k}$

(a)

(b)

Figure 10: Examples of photometric correction in the sequences (a) Planar Object and (b) Cylinder2 (point A) for different times $k$ : (1) without photometric correction. (2) correction with $P_{6}$. (3) Evolution of $\boldsymbol{\alpha}^{\top} \mathbf{u}$. (4) Evolution of $\boldsymbol{\beta}^{\top} \mathbf{u}$. 